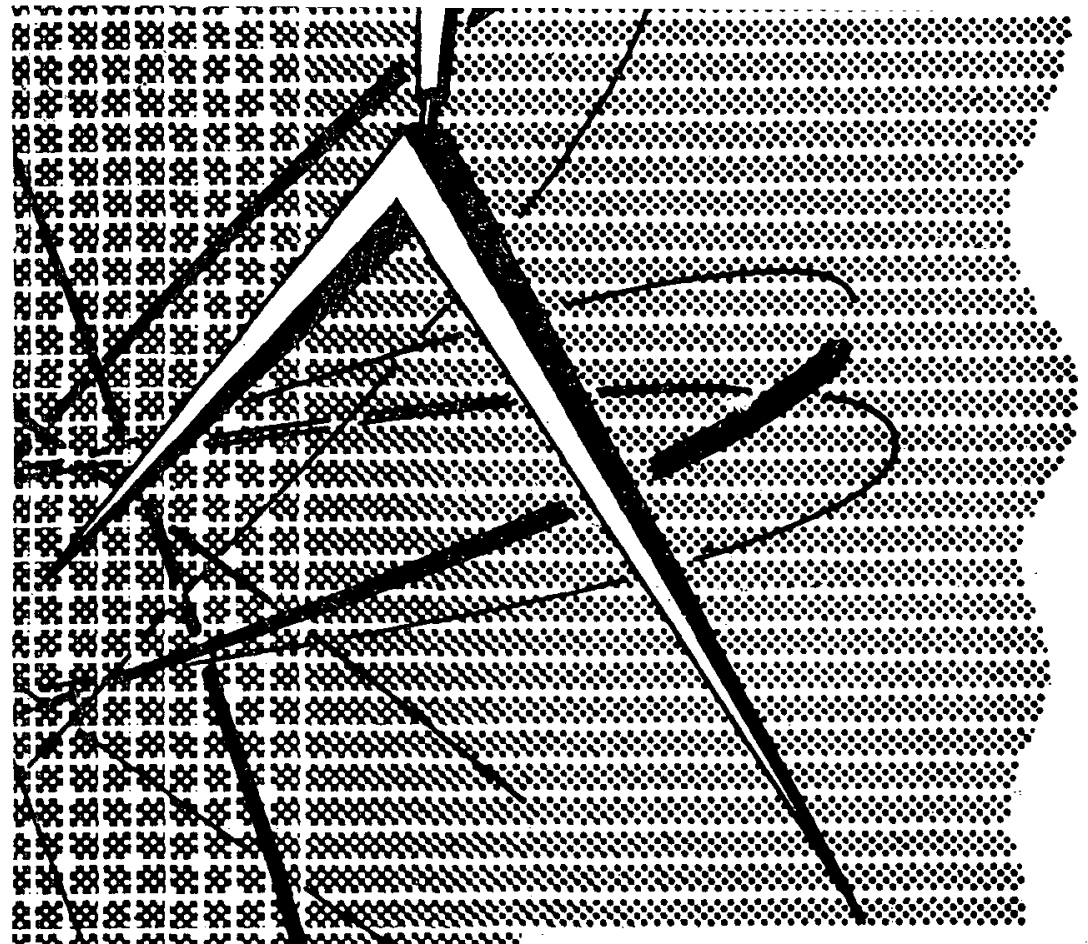

NDA 2147-3

MASTER

\title{
A STATUS REPORT ON REACTIVITY COEFFICIENTS IN FAST REACTORS AND METHODS OF INVESTIGATING THEIR EFFECTS ON REACTOR STABILITY
}

\author{
J. Agresta
}

March 1, 1961

\section{NDA -}

Work Performed under Contract AT(30-1)-2303 (XIII) for the United States Atomic Energy Commission

\section{NUCLEAR DEVELOPMENT CORPORATION OF AMERICA}




\section{DISCLAIMER}

This report was prepared as an account of work sponsored by an agency of the United States Government. Neither the United States Government nor any agency Thereof, nor any of their employees, makes any warranty, express or implied, or assumes any legal liability or responsibility for the accuracy, completeness, or usefulness of any information, apparatus, product, or process disclosed, or represents that its use would not infringe privately owned rights. Reference herein to any specific commercial product, process, or service by trade name, trademark, manufacturer, or otherwise does not necessarily constitute or imply its endorsement, recommendation, or favoring by the United States Government or any agency thereof. The views and opinions of authors expressed herein do not necessarily state or reflect those of the United States Government or any agency thereof. 


\section{DISCLAIMER}

Portions of this document may be illegible in electronic image products. Images are produced from the best available original document. 
NDA 2147-3

\title{
A STATUS REPORT ON REACTIVITY COEFFICIENTS IN FAST REACTORS AND METHODS OF INVESTIGATING THEIR EFFECTS ON REACTOR STABILITY
}

\author{
J. Agresta
}

Work Performed by:

J. Agresta

H. Soodak

March 1, 1961

Work Performed under Contract AT(30-1)-2303 (XIII) for the United States Atomic Energy Commission

NUCLEAR DEVELOPMENT CORPORATION OF AMERICA White Plains, New York 

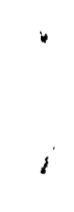

$\theta$ 


\section{CONTENTS}

1. INTRODUCTION AND SUMMARY . . . . . . . . . . . . . . . . . 1

2. ANALYTICAL METHODS ....................... 3

3. EXPERIMENTAL METHODS . . . . . . . . . . . . . . . . . . . . . 9

4. REACTIVITY COEFFICIENTS . . . . . . . . . . . . . . . . . 11

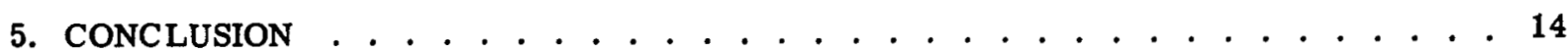

6. SELECTED BIBLIOGRAPHY . . . . . . . . . . . . . . . . 15

\section{TABLES}

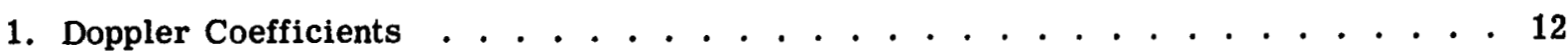

2. Isothermal Temperature Coefficients of Reactivity . . . . . . . . . . . . 12

\section{FIGURE}

1. Feedback System . . . . . . . . . . . . . . . . . . . 4 


\section{INTRODUCTION AND SUMMARY}

A major problem in the development of fast power breeder reactors is their stability and safety. This problem was emphasized by the meltdown accident of the Experimental Breeder Reactor (EBR-I) and is now receiving widespread attention in the legal difficulties associated with the completion of the Enrico Fermi Fast Breeder Reactor (EFFBR).

A point of early interest in fast reactor safety was the inherently short neutron lifetime in fast reactors. However, if the reactivity insertion is kept well below prompt critical, the delayed neutrons will dominate and the kinetics of a fast reactor will not differ radically from the kinetics of a thermal reactor.

Of greater interest in the safety of fast reactors is the absence of a single large negative temperature (or power) coefficient which will enhance stability. Instead, a fast reactor will usually have a number of small power coefficients some of which may be positive. In addition, the time response of these coefficients will be different because of the importance of various heat conduction and coolant transport delays.

If a reactor experiences a sudden increase in reactivity, it may respond by:

1. increasing power monotonically until destructive failure occurs,

2. increasing power and leveling off at some new equilibrium state,

3. oscillating in a convergent manner,

4. oscillating in a divergent manner until destructive failure occurs.

The response which does, in fact, take place, is determined by the interaction of the various reactivity coefficients, the magnitude of the initial reactivity insertion, and the nature of the heat removal system.

In general, a positive reactivity coefficient tends to render the reactor unstable. For this reason, the standard practice has been to design reactors with only negative reactivity coefficients. This imposes a limitation on reactor design which may make it impossible to achieve the goal of large, high capability, fast breeder reactors. A better understanding of the effects of small positive coefficients within an overall negative coefficient may remove this limitation and yet result in the design of stable and safe reactors.

An additional point of interest is that the absence of positive coefficients does not itself insure stability. In fact, a single negative coefficient which is delayed because of a coolant transport effect will result in instability at some power.

An investigation of the stability of fast reactors in the presence of both positive and negative reactivity coefficients with various response times constitutes part of a study of fast reactor safety problems for the United States Atomic Energy Commission under Contract AT(30-1)-2303 (XIII). The first phase of this investigation consisted of a survey to determine the status of methods 
of predicting stability of fast reactors in the presence of both positive and negative reactivity coefficients. The present report summarizes the results of this survey.

- The body of the report is divided into five general categories. The first part (Section 2) discusses analytical methods of predicting reactor stability. This includes discussions of the effects of "heat conduction" and "coolant transport" type delays in reactivity feedback. Stability criteria such as the Nyquist criterion and Welton's criterion are discussed and some general results presented.

The second part (Section 3) is concerned with experimental methods of determining reactor stability. The "excursion," "transient," and "oscillator" methods are discussed. Greatest emphasis is placed on the "oscillator" method in which the reactivity is oscillated (perhaps sinusoidally) and the power response observed as a function of oscillation frequency.

7 Section 4 discusses the sources of reactivity coefficients at both zero power and nonzero power operation. A general discussion of the effects peculiar to fast reactors is included. Some representative values of reactivity coefficients are also presented. The positive coefficients due to Doppler effect in highly-enriched reactors, fuel bowing, and sodium-coolant expansion in large plutonium reactors are discussed.

Section 5 contains an outline of suggested future work. This outline is based on the results of the survey which are briefly discussed below.

The survey indicates that many useful methods of predicting stability have been developed. However, virtually all applications have been made only to very special cases. A more general study of the influence of reactivity coefficients on fast reactor stability would better define the areas of stability and should indicate under what conditions, if any, positive reactivity coefficients are admissible. In addition, the applicability to fast reactors of generalized criteria (such as Welton's) has been largely neglected up to now and should be studied.

Section 6 contains a selected bibliography of important references. For each entry, a short summary of pertinent contents is included. 


\section{ANAL YTICAL METHODS}

In this report, we are concerned primarily with methods of predicting the stability of fast reactors under the influence of both positive and negative reactivity coefficients and with different time constants. One simple method of investigating the kinetic behavior of reactors is by digital or analog calculation of power or neutron flux as a function of time. This method has its experimental analogies in the so-called "transient" and "excursion" methods, the former characterized by small reactivity insertions and the latter by large insertions. In general, we will not consider the straightforward kinetic calculations but will be more concerned with stability criteria and analyses of a more general nature. It will be necessary to discuss both linear and nonlinear reactor behavior, the ultimate goal being the prediction of both linear and nonlinear stability.

A widely used method of investigating linear stability considers small oscillations about some equilibrium power level..$^{1,2,3}$ The general kinetics equations may be written as:

$$
\begin{aligned}
& \ell \frac{d n}{d t}=(\Delta k-1) n \beta+\sum_{i} \lambda_{i} C_{i} \\
& \frac{d C_{i}}{d t}=f_{i} n-\lambda_{i} C_{i}
\end{aligned}
$$

where $\mathrm{n}$ is the power level

$\Delta \mathrm{k}$ is the excess reactivity (in dollars)

$\ell$ is the prompt neutron lifetime

$\beta$ is the delayed neutron fraction

$C_{i}$ is the power contribution of the $i^{\text {th }}$ delayed group

$\lambda_{\mathbf{i}}$ is the $i^{\text {th }}$ group decay constant

$f_{i}$ is the $i^{\text {th }}$ group delay fraction.

Thus, e.g., if we consider an external reactivity oscillation of the form

$$
\Delta \mathrm{k}=\delta \mathrm{k} \mathrm{e}^{\mathrm{i} \omega \mathrm{t}}
$$

where $\Delta k=$ excess reactivity

$\omega=$ frequency of oscillation

then $\delta$, the amplitude of departure of power level from the equilibrium value $n_{0}$ is given by a relation of the form: ${ }^{1}$

$$
\frac{\delta \mathrm{n}}{\mathrm{n}_{0}}=\mathrm{G}(\mathrm{i} \omega) \delta \mathrm{k}
$$


where $G(i \omega)$ is the general reactor transfer function* and will be a function of time constants, delayed neutrons, neutron lifetime, reactivity feedback, etc. Eq. 3 is linearized, i.e., products of changes have been ignored.

For zero-power operation (no feedback), $G$ reduces to the zero-power transfer function which we will denote by $K(i \omega)$. For a feedback loop as indicated in Fig. 1, where $F B(i \omega)$ is the feedback term (assumed positive), $\mathrm{G}$ will be of the form ${ }^{1}$

$$
\mathrm{G}=\frac{\mathrm{K}}{1-\overline{\mathrm{KFB}}}
$$

A typical form ${ }^{1}$ of FB appropriate for prompt positive and delayed negative reactivity coefficients is:

$$
F B=n_{0}\left[\frac{\gamma_{f}}{1+\frac{i \omega}{\omega_{f}}}-\frac{\gamma_{S}}{\left(1+\frac{i \omega}{\omega_{f}}\right)\left(1+\frac{i \omega}{\omega_{S}}\right)}\right]
$$

where $f$ and $s$ respectively denote fast and slow response

$\gamma_{\mathbf{f}}$ and $\gamma_{\mathbf{S}}$ are the power coefficients of reactivity

$\omega_{\mathrm{f}}$ and $\omega_{\mathrm{S}}$ are appropriate response time constants $\left(\mathrm{sec}^{-1}\right)$.

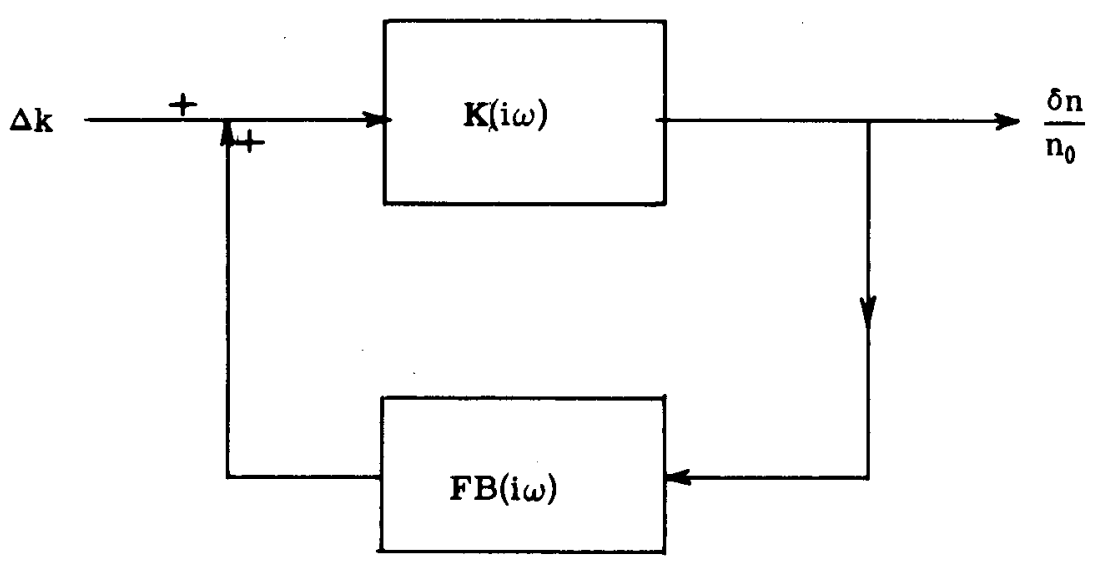

Fig. 1 - Feedback system

This form of FB is appropriate for the case where the delays are coupled conduction delays, e.g., the prompt delay is due to the heating of the fuel and coolant and the slow delay to the resulting heating of the structure. This form is not quite correct if there is also a transport delay between the two processes. This will, in general, require a definite time $\tau$ and may be included in Eq. 5 as a factor, $\mathrm{e}^{\mathrm{i} \omega \tau}$, multiplying the slow term. ${ }^{2}$

Eq. 3 gives the amplitude of the steady-state power vibrations about the original equilibrium level $n_{0}$. The time dependence of the oscillation will be $e^{i \omega t}$. In general, $G$ will be a complex number and thus supply both an amplitude and phase shift factor. The total time dependent solution will also contain transient terms of the form, $A_{i} e^{p_{i t}}$, where $p_{i}$ are characteristic roots of the homogeneous differential equation $[\Delta \mathrm{k}=0$ in (1)]. If these complex roots all have negative real

* The reactor transfer function for arbitrary forcing function $\Delta \mathrm{k}(\mathrm{t})$ is usually defined by $\overline{\delta n} / \mathrm{n}_{0}=$ $\mathrm{G}(\mathrm{s}) \overline{\delta \mathrm{k}}$ where the - indicates Laplace transform and $\mathrm{s}$ replaces $\mathrm{i} \omega$. In case of sinusoidal variation this reduces to Eq. 3 . 
parts, all of the transient terms will die out with time. If any one root has a positive real part, the transient term corresponding to it will build up with time and the system will be unstable. It may be shown ${ }^{1}$ that the characteristic equation defining these roots is:

$$
[G(s)]^{-1}=0
$$

Thus a means of investigating stability is by investigation of the real parts of the roots of Eq. 6 . In most typical cases, where five or more delayed neutron groups are considered, this will be a high order equation and solution for the roots difficult. Several methods are available for establishing the presence of roots with positive real parts without actually finding the roots. One such method $^{1,3}$ is the Nyquist Stability Criterion.* In circuit terminology, KFB is called the open loop gain. The open loop gain is plotted on the complex plane as $\omega$ varies from $-\infty$ to $+\infty$. For the system to be stable the curve must not enclose the point $-1+i 0$. This is equivalent to saying that there are no roots of the equation

$$
1-\mathrm{KFB}=0
$$

with positive real parts. In case the Nyquist plot passes through the point $-1+i 0$, then a resonant condition is present and the system is on the verge of instability. A study of this problem has been made by Siegel and Hurwitz ${ }^{1}$ for the case $1 / \omega_{\mathrm{f}}=0$ (no delay in prompt coefficient). For fixed values of the ratio of $\gamma_{\mathrm{s}} / \gamma_{\mathrm{f}}$, they have calculated as a function of $\omega$ values of $\omega_{\mathrm{s}}$ and corresponding values of $\gamma_{f} n_{0}$ corresponding to the borderline of instability. From these results, if $\gamma_{s}, \gamma_{f}, \omega_{S}$ are specified it is possible to find the power above which instability will occur. Some general conclusions of their results are:

1. Under some conditions, a combination of prompt positive plus delayed negative reactivity coefficients does not necessarily lead to instability.

2. As $\omega_{S}$ is increased (structure heats up more rapidly), the allowable values of $\gamma_{f} n_{0}$ increase.

3. As $\gamma_{S} / \gamma_{f}$ is reduced the system becomes less stable and for a given $\gamma_{f} n_{0}, \omega_{s}$ must be increased for the system to remain stable.

4. For $\gamma_{\mathbf{s}} / \gamma_{\mathrm{f}}<1$, the system is always unstable.

Bethe ${ }^{2}$, and Ackroyd et al., ${ }^{4}$ have made less general studies of this problem based on the EBR-I experimental behavior. There seems to be some confusion in these publications regarding the terms "resonance" and "instability" which are more or less used synonymously. It can be shown on the basis of a simple model that the mere presence of a resonance in an oscillator experiment does not necessarily indicate instability. Consider the linearized equation for $\Delta n$, neglecting delayed neutrons (or lumping prompt neutron lifetime and delay times into a single parameter $\ell$ )

$$
\frac{\partial \Delta \mathrm{n}}{\partial \mathrm{t}}=\frac{\Delta \mathrm{k}}{\ell} \mathbf{n}_{0}
$$

where $\Delta k$ is the excess reactivity and $\ell$ is the average neutron lifetime. Consider only a delayed negative feedback $\Delta \mathrm{k}_{\mathrm{FB}}$ which will satisfy an equation of the form

$$
\frac{1}{\omega_{S}} \frac{\partial \Delta k_{F B}}{\partial t}=-\Delta k_{F B}-b \Delta n
$$

* The statement of the Nyquist Theorem as made here is appropriate for the case with zero response at infinite frequency which is the usual reactor case. 
where $b$ is the negative reactivity power coefficient. For an oscillatory behavior all time variations go like $\mathrm{e}^{\mathrm{i} \omega \mathrm{t}}$. From Eq. 8

$$
\Delta \mathrm{k}_{\mathrm{FB}}=-\frac{\mathrm{b} \Delta \mathrm{n}}{\left(1+\mathrm{i} \frac{\omega}{\omega_{\mathrm{s}}}\right)}
$$

Since $\Delta \mathrm{k}=\left(\delta \mathrm{k}_{\mathrm{FB}}\right) \mathrm{e}^{\mathrm{i} \omega \mathrm{t}}$ and $\Delta \mathrm{n}=\delta \mathrm{n} \mathrm{e}^{\mathrm{i} \omega \mathrm{t}}$, from $\mathrm{Eq} .8$ it follows

$$
\frac{\delta \mathrm{n}}{\mathrm{n}_{0}}=\left[\frac{1+\frac{\mathrm{i} \omega}{\omega_{\mathrm{S}}}}{\left(\mathrm{n}_{0} \mathrm{~b}-\frac{\omega^{2} \ell}{\omega_{\mathrm{S}}}\right)+\mathrm{i} \omega \ell}\right] \delta \mathrm{k}=\mathrm{Z}(\mathrm{i} \omega) \delta \mathrm{k}
$$

The amplitude of $\mathrm{Z}(\mathrm{i} \omega)$ is then:

$$
|\mathrm{Z}|=\left[\frac{1+\left(\frac{\omega}{\omega_{\mathrm{s}}}\right)^{2}}{\left(\mathrm{n}_{0} \mathrm{~b}-\frac{\omega^{2} \ell}{\omega_{\mathrm{S}}}\right)^{2}+\omega^{2} \ell^{2}}\right]^{1 / 2}
$$

Eq. 12 is in a form which can yield a resonance for some value of $\omega$. However, it does not yield an infinity in the response and is thus not unstable. It can be shown that the characteristic roots of this simple system always have negative real parts and thus none of the transient terms will build up with time. A similar result is found in the work of Hurwitz and Siegel ${ }^{1}$ where the condition for stability, for small values of $\omega$, is

$$
\frac{\mathrm{n}_{0} \gamma_{\mathrm{f}}}{\mathrm{T} \omega_{\mathrm{S}}}<1
$$

where $\mathrm{T}$ is a function related to the neutron lifetime and delayed neutron parameters.

We may think of the response of the system to a step function of reactivity as the sum of responses to the different frequency Fourier components of the step. The final result is an overshoot and damped oscillation about a new equilibrium power. The magnitude of the overshoot will be determined by the resonance shape and even though the oscillations are damped the initial power surges may be excessive and dangerous.

The works of Bethe ${ }^{2}$ and Ackroyd et al., ${ }^{4}$ are primarily concerned with infinities in the response function and not with finite resonances. Thus, they define a "resonance" condition as one in which $\mathrm{Z}$ becomes infinite. They attempt to explain the observed resonance behavior of the EBR-I and approximately duplicate the resonances present using a combination of prompt positive and delayed negative reactivity coefficients. The general results of their studies are in agreement with Siegel and Hurwitz, ${ }^{1}$ namely that above a certain power a system as described above may be prone to instability.

A major difference between the work of Bethe and that of Siegel and Hurwitz is the inclusion by the former of a transport type delay. It is this type of delay which can lead to instability even in the absence of a positive reactivity coefficient. This can be shown from a simple analysis. Consider a feedback of the form (for oscillations with time dependence $e^{p t}$ )

$$
\Delta \mathrm{k}_{\mathrm{FB}}=-\mathrm{C} \mathrm{e}^{-\mathrm{p} \tau} \Delta \mathrm{N} \text {. }
$$


From the linearized power Eq. 8 it can be shown that the characteristic roots p satisfy

$$
\mathrm{p}+\frac{\mathrm{N}_{0} \mathrm{C}}{\ell} \mathrm{e}^{-\mathrm{p} \tau}=0
$$

$p$ is a complex number which may be written

$$
\mathrm{p}=\frac{1}{\tau}(\mathrm{u}+\mathbf{i} \nu)
$$

The equations satisfied by $u$ and $\nu$ are then

$$
\begin{aligned}
& \mathrm{u}+\frac{\mathrm{N}_{0} \mathrm{C} \tau}{\ell} \mathrm{e}^{-\mathrm{u}} \cos \nu=0 \\
& \nu-\frac{\mathrm{N}_{0} \mathrm{C} \tau}{\ell} \mathrm{e}^{-\mathrm{u}} \sin \nu=0 .
\end{aligned}
$$

From Eq. 17 it is possible to show that for an appropriate value of $\mathrm{N}_{0} \mathrm{C} \tau / \ell, \mathrm{u}$ is positive and the system is unstable even without a positive coefficient.

It can also be shown that two negative delays which are of the form (Eq. 5) can also lead to instability even if one is prompt.

Bethe emphasizes that the delayed negative coefficient is the prime factor determining the unstable resonance and the prompt positive coefficient only affects the details. However, the prompt positive coefficient is very important in the case of a rapid excursion where the delayed coefficient does not have time to act and may lead to a meltdown.

Sandmeier ${ }^{3}$ has used the Root-Locus method to study the stability of a fast reactor with parameters corresponding to the EBR-I. The Root-Locus is essentially a plot of the roots of the characteristic equation as a function of power level. If $\mathrm{K}$ and $\mathrm{FB}$ are specified as polynomials, this plot is relatively simple. By inspection of the Root-Locus, it is possible to trace the root behavior for different power levels and find the appearance of roots with positive real parts. The advantage of this method over the Nyquist diagram is the necessity of drawing only one curve instead of one for each power. The disadvantage is that for practical reasons $\mathrm{K}$ and FB must be given in terms of polynomials with known roots.

For large reactivity variations, the power variations, $\mathrm{n}$, will not be small compared to $\mathrm{n}_{0}$ and the linearized equations will not be valid. This will also be true for oscillation near a resonance where the amplification is high. This problem has been considered by Nelkin and Sandmeier. For the linear case, the response to a sinusoidal reactivity oscillation will also be sinusoidal and the transfer function is independent of the amplitude of the reactivity. In the nonlinear case, the transfer function will be amplitude-dependent and the response will no longer be sinusoidal. In the method considered here, the power variation is expanded in a Fourier series and the fundamental singled out and compared with the result from the linear case. If the fundamental oscillation has a higher amplitude than the linear oscillation for the same reactivity amplitude, it is concluded that the reactor is less stable for large disturbances. For the nonlinear case, it is necessary to oscillate the reactivity about some negative "bias" reactivity to avoid exponential increases and keep the average power constant. The value of this bias and the amplitudes of the Fourier components are obtained by an iterative procedure starting with the linear result as a first approximation. Some results found were that the EBR-I is less stable for large disturbances and that stability is enhanced for the case where the positive coefficient is limited (e.g., by clamping of bowing fuel elements). 
Several authors $\mathrm{s}^{5-9}$ have considered general stability criteria for generalized reactor systems. One such method ${ }^{6}$ uses a mechanical model as follows. A Hamiltonian is defined which when substituted into the usual Hamilton equations leads to appropriate reactor kinetics equations (which may be nonlinear). For example, the negative logarithm of power may be associated with a position coordinate, the temperature with a momentum coordinate, power with a force and a combination of various constant factors (e.g., power coefficient, neutron lifetime, heat capacity) with the mass. Forced periodic motion of the system is assumed and the energy balance studied. If on the average over a cycle, positive work is done by the driving force, then the nondriven system would be damped and the system is stable. Using this method Welton's criterion for unconditional nonlinear stability is expressed through the inequality:

$$
\int_{0}^{\infty} \mathrm{d} \tau K(\tau) \frac{\sin \omega \tau}{\omega}>0
$$

where $\tau$ is a time variable and

$$
\mathbf{K}(\tau)=-\frac{\dot{\mathrm{G}}(\tau)}{\mathrm{G}(0)}
$$

$\mathrm{G}(\tau)$ is a general kernel of the power coefficient of negative reactivity $\gamma$, so that at constant power

$$
\gamma=\int_{0}^{\infty} \mathrm{d} \tau \mathrm{G}(\tau)
$$

$\mathrm{G}(\tau)$ is the negative reactivity contribution at time $\tau$ arising from unit fission energy released in the reactor at time zero.

A similar study has been published ${ }^{9}$ recently using an electric circuit analog instead of a mechanical one. In this case, a periodic external voltage source is the driving force. If the power supplied by this external source is positive over a period, then the unforced circuit will be stable. The formulation is somewhat more concise than the mechanical formulation but the criterion obtained is equivalent. Consider the case where the feedback satisfies an equation of the form

$$
\delta k_{F B}(t)=\int_{-\infty}^{t} G\left(t-t^{\prime}\right) \delta N\left(t^{\prime}\right) d t^{\prime}
$$

where $G$ is a negative feedback kernel, the negative feedback reactivity at time $t$ due to a unit amount of excess integrated power at the previous time $t^{\prime}$ and is zero for $t^{\prime}>t$. Denoting Laplace transforms by - and transforming Eq. 20:

$$
\overline{\delta \mathrm{k}_{\mathrm{FB}}}=\overline{\mathrm{G}} \overline{\delta \mathrm{N}}
$$

Thus $\bar{G}(s)$ is the negative feedback transfer function. The sufficiency criterion for nonlinear stability takes the form

$$
\operatorname{Re}[\overline{\mathrm{G}}(\mathrm{i} \omega)] \geqslant 0 \quad \text { For }-\infty<\omega<\infty
$$

It can be shown ${ }^{9}$ that this is equivalent to Eq. 18. It should be pointed out that these general criteria are sufficient but not necessary for stability. 


\section{EXPERIMENTAL METHODS}

There are essentially three methods ${ }^{2}$ of investigating reactor stability: the excursion method, the transient method, and the oscillator method.

In the excursion method, a relatively large amount of reactivity is introduced and the power level measured as a function of time. For reactors with large prompt negative reactivity coefficients, this results in a rapid return to equilibrium. This type of experiment has been used many times at Los Alamos on such highly enriched assemblies as Godiva. ${ }^{10}$ One of the valuable results of these experiments is a check of analytical methods for predicting the total energy release in such an excursion. This is of interest in predicting what might happen in a power reactor following a meltdown which results in a sudden abnormal regrouping of the molten fuel. In general, an excursion experiment on a full scale reactor is dangerous and would yield little more information about instability than its presence. ${ }^{2}$ In the presence of a prompt positive reactivity coefficient, this method may easily lead to an accident. It was such an experiment on the EBR-I which led to its meltdown. ${ }^{11}$ The presence of a prompt positive coefficient had been indicated previously in experiments where the coolant flow was suddenly decreased or increased. The effect was larger at higher powers where larger temperature gradients were present resulting in the explanation that the effect was due to fuel rod bowing. It should be noted that in the presence of a prompt positive coefficient, a moderate amount of added reactivity is sufficient to cause an excursion.

The transient method is similar to the excursion method except that the added reactivity is relatively small (well below prompt critical). A prompt positive reactivity coefficient may easily be detected in a transient experiment at low or zero coolant flow. Introducing a small reactivity increase will then result in an exponential power rise. The presence of a resonance instability may also be found. As pointed out in the section on analytical methods, such instabilities will occur at some threshold power (for a fixed reactor design). Below this power a reactivity increase will cause damped oscillations about some new equilibrium power. However, in order to observe these damped oscillations the power must be relatively close to the instability threshold. In general, the sharper the resonance, the lower is the power needed to observe it. The transient method is not very useful in determining the frequency response especially far from the resonance. One method ${ }^{2}$ of doing this requires a Laplace analysis, i.e., an analysis of the observed power function into exponential components. In general, this is difficult and inaccurate especially at high power where the characteristic rate constants may not differ by much.

The best method of investigating instability and measuring power coefficients of reactivity is by controlled oscillator experiments. ${ }^{2}$ In such an experiment, the reactivity is oscillated (perhaps sinusoidally) and the power response observed as a function of oscillation frequency. From the experiment, the amplitude and phase of the response is obtained and the presence of resonances may be detected. In general, it will be necessary to perform these experiments over a wide range of powers since the effects may be nonlinear and the system may exhibit complicated hysteresis effects. The frequency response will be complex and both amplitude and phase must be measured. 
The phase is especially important in determining the power coefficient since, essentially, the power coefficient is obtained by vector subtraction of the zero power response function from the power response function. The amplitude is sufficient to give a rough idea about instabilities but the phase is required to determine the details about the power coefficient.

A prompt positive power coefficient can be measured more safely in an oscillator experiment than an excursion test. At zero (or greatly reduced) coolant flow, the time constant of the delayed coefficient will be greatly increased, effectively eliminating the delayed coefficient. Thus the measured frequency response will give information about the prompt coefficient. The power must be high enough so that the frequency response will be sufficiently different from the zero power response function.

In measuring the frequency response, care must be taken that the instability threshold power is not surpassed. Thus stepwise changes in power are required from experiment to experiment. From the maximum change in amplification it is possible to predict the next permissible step in power. ${ }^{2}$

Oscillator experiments were run on the EBR-I. ${ }^{11}$ Resonances were found which were functions of coolant flow and power. The shape of these resonances was reasonably well duplicated by a semiempirical method using the model discussed under analytical methods. In the EBR-I Mark-III core where great care was taken to eliminate bowing of fuel rods, instability appears to have been eliminated at all powers. This is reflected in the response function which contains no resonances. ${ }^{11}$ 


\section{REACTIVITY COE FFICIENTS}

In considering the sources of reactivity coefficients, it is necessary to differentiate between zero power and nonzero power operation. In the former case, the whole reactor complex is at essentially a uniform temperature and it is possible to consider changes in reactivity due to changes in temperature of individual components or due to a uniform change in temperature of the whole system. This type of coefficient is usually called the "temperature coefficient of reactivity," either partial or overall depending on whether partial or overall temperature changes are considered. The temperature coefficient of reactivity may be defined as $(\Delta k / k \Delta T)$ where $\Delta k$ is the reactivity change resulting from temperature change $\Delta T$. In case of partial coefficients, the $\Delta T$ is associated with a particular component.

In the case of operation at power, the reactivity coefficients will be more complex. In general, the temperature distribution in a reactor at power is not uniform and power changes may result in different changes in temperature at different locations in the reactor. The coefficient associated with such changes is usually called the "power coefficient of reactivity" and will, in general, depend on the operating conditions and particular reactor design. The power coefficient of reactivity may be defined as $(\Delta \mathrm{k} / \mathrm{k} \Delta \mathrm{n})$ where $\Delta \mathrm{k}$ is the reactivity change resulting from power change $\Delta \mathrm{n}$. An additional complication which must be considered in the case of power coefficients is the presence of time lags, e.g., a power change may result in rapid temperature changes in fuel and coolant followed by a slower temperature change in external structure. This relative time sequence can have a profound effect on stability of operation.

In fast reactors, the contributions to temperature and power coefficients of various components of the reactor are usually small with no single contribution overshadowing the others. ${ }^{12}$ In general then, the net coefficient will result from a competition between small negative and positive components and its sign and magnitude may depend on the structural details.

A discussion of various sources of reactivity coefficients in fast reactors follows.

In fast reactors, reactivity changes due to temperature-induced nuclear parameter changes are quite small. One such effect which has been widely studied is the Doppler effect. ${ }^{12-15}$ At fast and intermediate energies the capture and fission cross sections are composed of many closely spaced resonances. For a given neutron energy the relative energy of neutron and a nucleus is not fixed but is given by a distribution function due to the thermal motion of the nuclei. Changes in temperature tend to change the distribution, broadening it for temperature increase, sharpening it for temperature decrease. The effect of broadening this distribution is effectively to shorten the resonance peak and broaden the resonance width. The effect of the broadened resonance is relatively more important. If the resonance is predominantly a capture resonance, this will have a negative reactivity effect. If the resonance is predominantly a fission resonance, this will have a positive reactivity effect. In general, the resonances at high energies are already fairly wide and this effect is not as important as it would be for the more sharply peaked lower energy resonances. 
The Doppler effect can be calculated if the resonance structure of the cross sections is known. For energies of interest in fast reactors, this structure is not well known and estimates of resonance parameter distributions are necessary in order to make this calculation. Some general conclusions of these calculations for fast reactors ${ }^{14}$ are:

1. Pure $\mathrm{U}^{235}$ has a positive Doppler coefficient.

2. Pure $U^{238}$ has a negative Doppler coefficient.

3. A mixture of approximately equal numbers of atoms of $U^{238}$ and $U^{235}$ has a net zero Doppler coefficient.

Both calculated and measured values of Doppler coefficients have shown the effect to be small. Some examples are summarized in Table 1. There has been some question ${ }^{3}$ in interpretation of experimental results but, in general, it is agreed that the Doppler coefficient in fast reactors is too small to be of major importance. However, a positive Doppler coefficient furnishes a prompt coefficient and will contribute to instability. In large fast reactors with moderate enrichment, the Doppler coefficient will be negative and in some cases may be sufficient to slow down prompt excursions. ${ }^{14}$

Table 1 - Doppler Coefficients

\begin{tabular}{llll}
\multicolumn{1}{c}{ Example } & \multicolumn{1}{c}{ Values (dk/dt) } & Enrichment & Reference \\
& & & \\
EBR-I* (calc.) & $+2 \times 10^{-6} /{ }^{\circ} \mathrm{C}$ (Room temp) & Fully enriched & 12 \\
EFFBR† (calc.) & $-1.9 \times 10^{-6} /{ }^{\circ} \mathrm{C}$ & $\sim 25 \% \mathrm{U}^{235}$ & 12 \\
EBR-I mockup in & $<+1.6 \times 10^{-6} /{ }^{\circ} \mathrm{C}$ & Fully enriched & 31 \\
ZPR-III (meas.) & & $\sim 50 \% \mathrm{U}^{235}$ & 16 \\
EBR-II (calc.) & $+4 \times 10^{-7} /{ }^{\circ} \mathrm{C}$ & \\
& &
\end{tabular}

An important effect of temperature changes in fast reactors results from the changes in density and geometry of the various components. This affects both the relative sizes of components and the neutron leakage and may result in both positive and negative reactivity effects. Consider, for example, the expansion of fuel rods in a reactor. The decreased density results in a decrease of macroscopic cross sections and usually a negative reactivity effect. The increased size results in a larger effective core which contributes a positive reactivity effect. In general, the most important effect of density decreases (of all components) is an increase of leakage, a net negative reactivity effect. The decrease in density of structure and coolant also leads to a decrease in parasitic capture, a positive reactivity effect. For fast neutrons, this is frequently not a significant effect. A summary of some representative temperature coefficients is shown in Table 2.

Table 2 - Isothermal Temperature Coefr:a nts of Reactivity ${ }^{12}$

Mechanism

$$
\text { Core }
$$

Axial fuel expansion

Radial fuel expansion (Na expulsion)

Density change of coolant and subassembly material

Blanket

Structure expansion

Density change of coolant and subassembly

material

Growth of uranium

Structure expansion

$$
\frac{\Delta \mathrm{k}}{\mathrm{k} /{ }^{\circ} \mathrm{C}} \times 10^{+8}
$$

EBR-II $\quad$ EFFBR

$\begin{array}{ll}-3.9 & -2.5 \\ -0.9 & -0.6 \\ -9.1 & -7.1 \\ -9.7 & -6.0 \\ -9.5 & -3.3 \\ -1.0 & -0.5 \\ -2.0 & -0.6\end{array}$


An important special feature of fast reactors is their relatively small core size. This is one of the reasons why small changes in density affect primarily the neutron leakage. This is apparent from Table 2 where it is seen that the coefficients are generally larger in magnitude for the EBR-II which is smaller in size than the EFFBR.

An average power coefficient may be obtained by calculating an equilibrium temperature distribution at power and from this, the average temperature of each component. Then the partial temperature coefficients for these components may be suitably combined to give an overall average power coefficient. A power coefficient calculated in this manner is a "static" coefficient and, in general, would not be correct for rapid transients which do not allow quasi-static equilibrium. In such cases, the time of response must be accounted for.

Most stability studies are based on sinusoidal power oscillations. In such studies, time delays are easily accounted for by suitable phase shifts in the appropriate feedback terms. The sources of time delays are usually heat transfer or transport effects, e.g., it is expected that power changes will first affect the temperature of the fuel. This will then result in changes of coolant temperature as well as possible coolant expulsion due to fuel expansion. In addition, the coolant will transport heat out of the core and change the temperature of the outer portions of the reactor. This latter effect is of great importance since a delayed negative reactivity coefficient may result in a resonance.

An important reactivity coefficient which results from nonuniform temperature distributions is caused by bowing or buckling of components, ${ }^{2}$ especially fuel. Consider fuel rods which are supported only at both ends. The radial temperature will be higher near the core axis and will tend to bow the rods inward because of the greater expansion of the inner portion due to the higher temperature there. This bows fuel in a direction of greater importance and results in a reactivity increase. An effect as described here would cause a prompt positive coefficient which would be significant in fast transients and also would contribute to resonance instability. The problem of fuel bowing has been long recognized and current designs attempt to eliminate (or limit) it by positive mechanical means. ${ }^{11}$

In case the fuel is contained as small pins in subassemblies, it is the bowing of the whole subassemblies which must be considered. In general, this would not occur as rapidly as fuel bowing (due to heat transfer delays from fuel to subassembly) and may result in a net positive slow coefficient.

In general, the time response and magnitude of power coefficients will depend on actual details of reactor design, power level of operation, and coolant flow rate.

A reactivity coefficient which is found in large plutonium-fueled reactors is a prompt positive coolant coefficient caused by spectral changes. ${ }^{30}$ In uranium-fueled reactors, the major effect of coolant (e.g., sodium) density decreases is an increase of leakage, a negative reactivity effect. There is also a spectrum hardening which affects the relative average fission cross section compared with average absorption cross sections of other elements present. The fission cross section of plutonium is relatively insensitive to spectral changes and thus spectrum hardening can result in a positive reactivity effect. In some cases this may override the leakage effect, resulting in a net positive reactivity coefficient. Since this involves only heat transfer to the coolant, this coefficient will be relatively prompt and may contribute to instability. This effect will be less important in smaller reactors because of the relatively greater importance of leakage. 


\section{CONCLUSION}

A general conclusion of this survey is that many useful methods of predicting stability have been developed. However, virtually all applications have been made only to very special cases. A systematic investigation of the effect on fast reactor stability of various reactivity coefficients will be of value. Existing methods of analysis will probably be sufficient to carry out the plan outlined below. Such a program will include:

1. Single negative delayed coefficient.

a. Conduction type; investigation of resonances as a function of various reactor parameters; correlation of power overshoot with resonances.

b. Convection type; investigation of stability as a function of reactor parameters.

2. Negative delayed coefficient in combination with a prompt coefficient.

a. Conduction type delay; investigation of effects on resonances and instabilities of both positive and negative prompt coefficients.

b. Convection type delay; investigation of effects on resonances and instabilities of both positive and negative prompt coefficients.

c. Combined conduction and convection type delay.

3. In all cases, applicability of generalized stability criteria (such as Welton's) will be investigated. 


\section{SELECTED BIBLIOGRAPHY}

1. R. Siegel and H. Hurwitz, Jr., The Effect of Temperature Coefficients on Reactor Stability and Reactor Transfer Function, KAPL-1138 (1955).

General stability considerations; Nyquist criterion; transfer functions; parameter survey for combination of prompt positive and delayed negative reactivity coefficients; appendix by Nelkin on nonlinear effects; determination of reactivity coefficient from oscillator experiments.

2. H. Bethe, Reactor Safety and Oscillator Tests, APDA-117 (1956).

Discussion of transient, excursion, and oscillator tests; analysis of EBR-I oscillations; prompt positive coefficient; instability due to delayed negative coefficient; analysis of transient and oscillator experiments to determine power coefficients; feedback models for conduction and transport delays.

3. H. A. Sandmeier. The Kinetics and Stability of Fast Reactors with Special Consideration of Nonlinearities, ANL-6014 (1959).

Linearized zero-power kinetics and transfer functions; linear stability; Root-Locus method; Nyquist criterion; calculation of reactivity power coefficients; nonlinear effects by the incremental kinetics equations; limited bowing; nonlinear stability; analog com puter methods for fast reactors.

4. R. T. Ackroyd, G. H. Kinchin, J. E. Mann, and J. D. McCullen, Stability Considerations in the Design of Fast Reactors, PICG-12, P/1462 (1958).

Discussion of temperature and power coefficients of reactivity; oscillatory behavior with reactivity feedback; instability; feedback models for conduction delays; analysis of EBR-I oscillations; solutions for step-change and ramp-change of reactivity; analog computer results for various reactivity changes.

5. T. A. Welton, Kinetics of Stationary Reactor Systems, PICG-5, P/610 (1955).

General discussion of linear and nonlinear stability; mechanical analog of reactor system; Hamiltonian formulation; derivation of criterion for linear stability and application to several examples; derivation of criterion for unconditional nonlinear stability.

6. W. K. Ergen and A. M. Weinberg, Some Aspects of Nonlinear Reactor Dynamics, Physica 20:413 (1954).

Stability study using Hamiltonian formulation; application to adiabatic case, Newton's law of cooling, constant power removal, circulating fuel reactor, general delayed reactivity coefficient; generalized nonlinear kinetics.

7. W. K. Ergen, Kinetics of the Circulating Fuel Nuclear Reactor, J. Appl. Phys. 25:702 (1954). Hamiltonian formulation; application of Welton's stability criterion.

8. E. Gyftopoulos and J. Devooght, Boundedness and Stability in Nonlinear Reactor Dynamics, Nuclear Sci. and Eng. 7:533 (1960).

General discussion of stability; derivation of a stability criterion equivalent to Welton's; application to circulating fuel and boiling water reactors. 
9. A. Z. Akcasu and A. Dalfes, A Study of Nonlinear Reactor Dynamics, Nuclear Sci. and Eng. $8: 89(1960)$.

General discussion of nonlinear stability; use of electrical analogy; stability criterion equivalent to Welton's.

10. W. R. Stratton, T. H. Cowin, and R. B. Lazarus, Analysis of Prompt Excursions in Simple Systems and Idealized Fast Reactors, PICG-12, P/431 (1958).

Description of computer code to calculate prompt excursions in simple fast reactors; application to Godiva and similar assemblies; analysis of bursts using Bethe-Tait method; discussion of meltdown accidents; comparison of analytical methods.

11. F. W. Thalgott et al., Stability Studies on EBR-I, PICG-12, P/1845 (1958).

Prompt positive power coefficient; transfer functions and resonances; good discussion of feedback models; comparison of Mark I, II, III; effect of fuel clamping on stability; experimental techniques; oscillator test results; analytical methods of calculating transfer functions; effects on stability of various reactivity coefficients.

12. W. J. McCarthy Jr., R. B. Nicholson, D. Okrent, and V. F. Jankus, Studies of Nuclear Accidents in Fast Power Reactors, PICG-12, P/2165 (1958).

Good discussion of sources of temperature and power coefficients; Doppler effect; examples of possible accidents; discussion of core meltdown and effects.

13. Fast Reactor Control and Safety Meeting, APDA-105 (1954). EBR experience; Doppler effect; general fast reactor kinetic studies; prompt excursions in simple systems; oscillator tests.

14. R. B. Nicholson, The Doppler Effect in Fast Neutron Reactors, APDA-139 (1960). Lengthy discussion of Doppler effect; methods for calculation of Doppler coefficient of reactivity, effective cross sections; discussion of theoretical cross sections; resonance level spacing and widths; calculations for $\mathrm{U}^{235}$ and $\mathrm{U}^{238}$; suggestions for future work.

15. Proceedings of the Fast Reactor Information Meeting, M-7148 (1957).

Discussion of EBR-I; meltdown accidents.

16. Koch, EBR-II Hazard Report, ANL-5719

Hazard summary report; reactivity coefficients.

17. G. Kinchin, The Stability of Fast Reactors, AERE RP/M-83 (1956).

Discussion of EBR-I instability; positive coefficient due to rod bowing; calculation of resonances using prompt positive and delayed negative feedback model; effect of different coolant flows.

18. R. O. Brittan, Some Problems in the Safety of Fast Reactors, ANL-5577.

Discussion of computer kinetics codes; test problems for excursion type studies; discussion of EBR-I transfer function and instabilities; analytical solutions for various kinetics problems.

19. G. H. Kinchin, Kinetics of the Dounreay Fast Reactor, AERE RP/M-104 (1956). Kinetic study using reactor simulator; bowing coefficient; results for various operating conditions.

20. J. R. Dietrich, EBR Oscillations, TID-5351 (1956).

Discussion of EBR oscillations based on simple thermal lags; discussion of temperature and power coefficients.

21. A. J. UIrich, EBR-I Oscillations: Infinities in the Transfer Function with Positive and Negative Thermal Feed-Back for Sinusoidal Power Variation, TID-5352 (1956).

Calculation of instabilities in EBR-I; frequency of resonance for various time delays.

22. EBR Progress Report, ANL-5023 (1953).

Startup and operational experience; low power temperature coefficient; prompt positive fuel coefficient.

23. J. F. Boland, R. R. Smith, R. E. Rice, A Measurement of the Transfer Function of a Fast Critical Assembly, ANL-5782 (1957).

Mockup of EBR-I; measurement of amplitude and phase of zero power frequency response; comparison with theory. 
24. J. E. Mann, A Note on EBR-I Oscillations, IGR-TM/R-114 (1957).

Calculation of resonances found in EBR-I.

25. H. Brown and W. Loewe, Analysis of Reactor Oscillations for Coefficients of Reactivity, Nuclear Sci. and Eng., 5:376 (1959).

Technique of measuring reactivity coefficients in large reactors from oscillator tests; nonsinusoidal os cillations.

26. G. Brown and D. Campbell,"Principles of Servomechanisms," J. Wiley and Sons, New York, 1948.

Stability of feedback systems; frequency response; Nyquist criterion.

27. J. G. Truxal, "Control System Synthesis," McGraw-Hill Book Company, New York, 1955.

Stability of feedback systems; frequency response; Root-Locus methods.

28. M. A. Schultz, "Control of Nuclear Reactors and Power Plants," McGraw-Hill Book Company, New York, 1955.

General discussions of reactor systems control; feedback systems; frequency response; Nyquist criterion.

29. J. MacPhee, The Stability of Multi-Pass Reactors, Nuclear Sci. and Eng., 4:200 (1958).

Stability for system with two distinct temperature coefficients; Nyquist criterion; ranges of parameters for stability.

30. J. B. Nims and P. F. Zweifel, Preliminary Report on Sodium Temperature Coefficients in Large Fast Reactors, APDA-135 (1959).

Calculation of coolant temperature coefficients in large fast reactors with different fuels; spectral effects; discussion of causes of various results; recommendations for reducing prompt positive reactivity coefficient.

31. R. Frost, W. Kato and D. Butler, Measurement of the Doppler Temperature Coefficient in Intermediate and Fast Assemblies, PICG-12, P/1777 (1958).

Experimental studies of Doppler coefficient; results for various fuels; comparison with theory. 


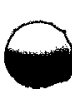




\section{DISTRIBUTION}

No. of

Copies

United States Atomic Energy Commission

New York Operations Office

John D. Hart, Contracts Division . . . . . . . . . . . . . . . . . . . . 1

Jules Wise, Reactor Division . . . . . . . . . . . . . . . . . . 3

Division of Reactor Development

Sodium Reactors Branch . . . . . . . . . . . . . . . . . . 25

E. E. Hall, Reports and Statistics Branch . . . . . . . . . . . . . . 3

Patents Group

Harmon Potter . . . . . . . . . . . . . . . . . . . 1

Technical Information Service Extension . . . . . . . . . . . . . . . 3 